\title{
A diophantine inequality involving prime powers
}

\author{
by
}

\author{
A. Kumchev (Columbia, SC)
}

1. Introduction. In 1952 I. I. Piatetski-Shapiro [8] studied the inequality

$$
\left|p_{1}^{c}+\ldots+p_{s}^{c}-N\right|<\varepsilon
$$

where $c>1$ is not an integer, $\varepsilon$ is a fixed small positive number, and $p_{1}, \ldots, p_{s}$ are primes. He established the existence of an $H(c)$, depending only on $c$, such that for all sufficiently large real $N,(1.1)$ has solution whenever $s \geq H(c)$. He proved that

$$
\limsup _{c \rightarrow \infty} \frac{H(c)}{c \log c} \leq 4
$$

and also that $H(c) \leq 5$ if $1<c<3 / 2$. On the other hand, the VinogradovGoldbach theorem [11] suggests that at least for $c$ close to 1 , one should expect $H(c) \leq 3$. The first result in this direction was obtained by $\mathrm{D}$. I. Tolev [10], who showed that the inequality

$$
\left|p_{1}^{c}+p_{2}^{c}+p_{3}^{c}-N\right|<\varepsilon
$$

with $\varepsilon=N^{-(1 / c)(15 / 14-c)} \log ^{9} N$ is solvable in primes $p_{1}, p_{2}, p_{3}$, provided that $1<c<15 / 14$ and $N$ is sufficiently large. Later this result was improved by Y. C. Cai [1] who replaced $15 / 14$ by $13 / 12$, and by T. Nedeva and the author [5] who obtained the range $1<c<11 / 10$. In this paper we give a further improvement, changing the upper bound for $c$ to $61 / 55$ (this constant can be improved somewhat, but not substantially; even 1.11 seems to be out of the scope of the method). For comparison

$$
\begin{array}{ll}
15 / 14=1.071428 \ldots, & 13 / 12=1.083333 \ldots, \\
11 / 10=1.100000 \ldots, & 61 / 55=1.109090 \ldots
\end{array}
$$

1991 Mathematics Subject Classification: Primary 11P32, 11P55; Secondary 11N36.

Research was done as partial fulfillment of the Ph.D. requirement at the University of South Carolina. 
The results in [1] and [5] are based on the version of the circle method used by Tolev enhanced by sharper exponential sum estimates. This approach, however, does not allow much further improvement, as a closer look at [5] shows. So, we combine it with Harman's sieve [3, 4], which allows more flexible use of the available arithmetical information. The essence is to apply Vinogradov's method [11] to the arithmetical problem itself rather than to the exponential sums arising from the analytic part of the argument. This allows us to discard some awkward cases that needed to be treated before. As a result we obtain a lower bound for the number of solutions instead of the asymptotic formula given by the previous approach. We prove

Theorem 1. Let $c$ be fixed with $1<c<61 / 55$ and $\delta>0$ be a fixed number sufficiently small in terms of $c$. Let also $N$ be a sufficiently large real number, and $\varepsilon \geq N^{-(1 / c)(61 / 55-c+\delta)}$. Then the number $R(N)$ of the solutions of (1.2) satisfies

$$
R(N) \gg \frac{\varepsilon N^{3 / c-1}}{\log ^{3} N} .
$$

The implied constant depends only on $c$.

A natural question to ask is what the "ideal" result should be. We give a probabilistic argument which suggests that one should expect $H(c) \leq 3$ at least for $1<c<3 / 2$. The theorem we prove is as follows:

Theorem 2. For $1<c<3 / 2$, let

$$
\varepsilon_{0}(c)=N^{-(1 / c)(3 / 2-c)} \log ^{10} N .
$$

Then for almost all (in the sense of Lebesgue measure) values of $c \in(1,3 / 2)$, the inequality (1.2) is solvable for $\varepsilon \geq \varepsilon_{0}(c)$ and sufficiently large values of $N$.

Notation. Throughout the paper $p, q, r$, indexed or not, always denote primes; $d, k, l, m, n$ denote integers. We choose $X=\frac{1}{4} N^{1 / c}$; in Sections $2-5, \eta=\delta^{2}$ where $\delta$ is the number from the statement of Theorem 1. Also, $m \sim M$ means that $m$ runs through the interval $(M, 2 M]$ and $e(x)=e^{2 \pi i x}$; $\varphi(y)$ is a function having $r=[\log X]$ continuous derivatives and the following properties:

1) $\varphi(y)=1$ for $|y| \leq 9 \varepsilon / 10$,

2) $\varphi(y)=0$ for $|y| \geq \varepsilon$,

3) $0<\varphi(y)<1$ for $9 \varepsilon / 10<|y|<\varepsilon$,

4) its Fourier transform

$$
\Phi(x)=\int_{-\infty}^{\infty} \varphi(y) e(-x y) d y
$$

satisfies the inequality 


$$
|\Phi(x)| \leq \min \left(2 \varepsilon, \frac{1}{\pi|x|}, \frac{1}{\pi|x|}\left(\frac{5 r}{\pi \varepsilon|x|}\right)^{r}\right) .
$$

One can construct it using Lemma 1 of [8].

Finally, throughout the proof of Theorem 1 we assume, as we can, that $\varepsilon=N^{-(1 / c)(61 / 55-c+\delta)}$. Similarly, in Section $6, \varepsilon=\varepsilon_{0}(c)$.

2. The sieve method. We write

$$
P(z)=\prod_{p<z} p
$$

and, as usual, for any sequence $\mathcal{E}$ of integers weighted by the numbers $w(n)$, $n \in \mathcal{E}$, we set

$$
S(\mathcal{E}, z)=\sum_{\substack{n \in \mathcal{E} \\(n, P(z))=1}} w(n),
$$

and denote by $\mathcal{E}_{d}$ the subsequence of elements $n \in \mathcal{E}$ with $n \equiv 0(\bmod d)$. So, if we define $\mathcal{A}$ to be the sequence of integers $n \in(X, 2 X]$ weighted by

$$
w(n)=\sum_{p_{1}, p_{2} \sim X} \varphi\left(p_{1}^{c}+p_{2}^{c}+n^{c}-N\right)
$$

we will have

$$
R(N) \geq \sum_{p_{1}, p_{2}, p_{3} \sim X} \varphi\left(p_{1}^{c}+p_{2}^{c}+p_{3}^{c}-N\right)=S\left(\mathcal{A},(3 X)^{1 / 2}\right) .
$$

Hence, it suffices to show that

$$
S\left(\mathcal{A},(3 X)^{1 / 2}\right) \gg \frac{\varepsilon X^{3-c}}{\log ^{3} X} .
$$

We prove (2.1) using the Buchstab identity

$$
S\left(\mathcal{E}, z_{1}\right)=S\left(\mathcal{E}, z_{2}\right)-\sum_{z_{2} \leq p<z_{1}} S\left(\mathcal{E}_{p}, p\right)
$$

and asymptotic formulas of the form

$$
\sum_{m \sim M} a(m) S\left(\mathcal{A}_{m}, z(m)\right)=\lambda \sum_{m \sim M} a(m) S\left(\mathcal{B}_{m}, z(m)\right)+\text { error terms }
$$

where $\mathcal{B}$ is the set of integers in $(X, 2 X]$, and $\lambda, M$, and $z(m)$ are appropriately chosen. The idea is to use $(2.2)$ to represent $S\left(\mathcal{A},(3 X)^{1 / 2}\right)$ as the linear combination of sums of the form appearing on the left-hand side of (2.3) so that we are able to give asymptotic formulas for all sums having a negative contribution to $S\left(\mathcal{A},(3 X)^{1 / 2}\right)$, as well as for most of those with a positive contribution. If it happens that the positive sums prevail, discarding the remaining positive terms, we get a positive lower bound. 
Throughout the rest of this section we set up the decomposition. We set $A=X^{89 / 825}, B=X^{12 / 55}, C=X^{844 / 3025}, D=X^{56 / 165}$, and $F=X^{123 / 275}$. Applying (2.2), we find

$$
\begin{aligned}
S\left(\mathcal{A},(3 X)^{1 / 2}\right)= & S(\mathcal{A}, A)-\sum_{A \leq p<B} S\left(\mathcal{A}_{p}, p\right)-\sum_{B \leq p \leq C} S\left(\mathcal{A}_{p}, p\right) \\
& -\sum_{C<p<D} S\left(\mathcal{A}_{p}, p\right)-\sum_{D \leq p \leq F} S\left(\mathcal{A}_{p}, p\right)-\sum_{F<p<\sqrt{3 X}} S\left(\mathcal{A}_{p}, p\right) \\
= & S_{1}-S_{2}-S_{3}-S_{4}-S_{5}-S_{6}, \quad \text { say. }
\end{aligned}
$$

We give a further decomposition for $S_{2}$ and $S_{4}$. Another application of (2.2) gives

$$
\begin{aligned}
S_{2}= & \sum_{A \leq p<B} S\left(\mathcal{A}_{p}, A\right)-\sum_{\substack{A \leq q<p<B \\
p q<B}} S\left(\mathcal{A}_{p q}, q\right) \\
& -\sum_{\substack{A \leq q<p<B \\
B \leq p q \leq C}} S\left(\mathcal{A}_{p q}, q\right)-\sum_{\substack{A \leq q<p<B \\
C<p q<D}} S\left(\mathcal{A}_{p q}, q\right)-\sum_{\substack{A \leq q<p<B \\
p q \geq D}} S\left(\mathcal{A}_{p q}, q\right) \\
& =S_{7}-S_{8}-S_{9}-S_{10}-S_{11}, \quad \text { say. }
\end{aligned}
$$

Similarly, we obtain

$$
\begin{aligned}
S_{4}= & \sum_{C<p<D} S\left(\mathcal{A}_{p}, A\right)-\sum_{\substack{C<p<D \\
A \leq q<B}} S\left(\mathcal{A}_{p q}, q\right) \\
& -\sum_{\substack{C<p<D \\
B \leq q \leq C}} S\left(\mathcal{A}_{p q}, q\right)-\sum_{\substack{C<q<p<D\\
}} S\left(\mathcal{A}_{p q}, q\right) \\
= & S_{12}-S_{13}-S_{14}-S_{15}, \quad \text { say. }
\end{aligned}
$$

Now, we deal with $S_{6}$. It counts numbers of the form $p q$, namely

$$
S_{6}=\sum_{\substack{p q \sim X \\ F<p \leq q}} w(p q) .
$$

It turns out to be more convenient to switch the sifting process from the product $p q$ to one of the primes $p_{1}, p_{2}$ from the definition of $w(n)$, say $p_{2}$. In order to do so, we write $S_{6}$ as $S\left(\mathcal{A}^{*},(3 X)^{1 / 2}\right)$ where $\mathcal{A}^{*}$ is the set of integers in $(X, 2 X]$ weighted by

$$
w^{*}(n)=\sum_{p_{1} \sim X} \sum_{\substack{p q \sim X \\ F<p \leq q}} \varphi\left(p_{1}^{c}+n^{c}+(p q)^{c}-N\right) .
$$

Let $S_{i}^{*}$ denote a sum similar to $S_{i}$ in which $\mathcal{A}$ has been replaced by $\mathcal{A}^{*}$. We decompose $S\left(\mathcal{A}^{*},(3 X)^{1 / 2}\right)$ following the same lines without decomposing $S_{4}^{*}$ and $S_{6}^{*}$. 
Putting all together and using that $S_{8}, S_{10}, S_{4}^{*}$, and $S_{6}^{*}$ have positive contributions to $S\left(\mathcal{A},(3 X)^{1 / 2}\right)$, we obtain

$$
\begin{aligned}
S\left(\mathcal{A},(3 X)^{1 / 2}\right) \geq & S_{1}-S_{3}-S_{5}-S_{7}+S_{9}+S_{11}-S_{12}+S_{13}+S_{14} \\
& +S_{15}-S_{1}^{*}+S_{3}^{*}+S_{5}^{*}+S_{7}^{*}-S_{8}^{*}-S_{9}^{*}-S_{10}^{*}-S_{11}^{*} .
\end{aligned}
$$

We will be able to find asymptotic formulas for all of these except for $S_{13}$, $S_{15}, S_{8}^{*}$, and $S_{10}^{*}$. Also, we will find asymptotic formulas for parts of $S_{13}$ and $S_{15}$ as well as admissible upper bounds for $S_{8}^{*}$ and $S_{10}^{*}$.

3. Exponential sums. In this section we prove the exponential sum estimates we will need to get the asymptotic formulas (2.3).

Lemma 1. Let $\alpha$ and $\beta$ be real, $\alpha \beta(\alpha-1)(\beta-1)(\alpha-2)(\beta-2) \neq 0$, $X>0, M, N \geq 1,|a(m)| \leq 1,|b(n)| \leq 1$. Then

$$
\begin{aligned}
(X M N)^{-\eta} & \left|\sum_{m \sim M} \sum_{n \sim N} a(m) b(n) e\left(X \frac{m^{\alpha} n^{\beta}}{M^{\alpha} N^{\beta}}\right)\right| \\
\ll & \left(X^{4} M^{31} N^{34}\right)^{1 / 42}+\left(X^{6} M^{53} N^{51}\right)^{1 / 66}+\left(X^{6} M^{46} N^{41}\right)^{1 / 56} \\
& +\left(X^{2} M^{38} N^{29}\right)^{1 / 40}+\left(X M^{9} N^{6}\right)^{1 / 10}+\left(X^{2} M^{7} N^{6}\right)^{1 / 10} \\
& +\left(X M^{6} N^{6}\right)^{1 / 8}+M^{1 / 2} N+M N^{1 / 2}+X^{-1 / 4} M N .
\end{aligned}
$$

Proof. This is Theorem 9 of [9]. that

In the following lemma and its applications $f(x, y) \sim_{\Delta} g(x, y)$ means

$$
\frac{\partial^{i+j}}{\partial x^{i} \partial y^{j}} f(x, y)=\frac{\partial^{i+j}}{\partial x^{i} \partial y^{j}} g(x, y)(1+O(\Delta))
$$

for all pairs $(i, j)$ for which this makes sense.

Lemma 2. Let $\mathcal{D}$ be a subdomain of the rectangle

$$
\{(x, y): M<x \leq 2 M, N<y \leq 2 N\}
$$

$(M \geq N)$ such that any line parallel to any coordinate axis intersects it in $O(1)$ line segments. Let $\alpha, \beta$ be real numbers, $\alpha \beta(\alpha+\beta-1)(\alpha+\beta-2) \neq 0$, and let $f(m, n)$ be a real sufficiently many times differentiable function such that $f(m, n) \sim_{\Delta} A m^{\alpha} n^{\beta}$ throughout D. Setting $X=M N, F=A M^{\alpha} N^{\beta}$, we have

$$
\begin{aligned}
(X F)^{-\eta}\left|\sum_{(m, n) \in \mathcal{D}} e(f(m, n))\right| \ll & \left(F^{2} X^{3}\right)^{1 / 6}+X N^{-1 / 2}+X^{5 / 6} \\
& +X\left(\Delta M^{-1}\right)^{1 / 4}+X(F M)^{-1 / 8} \\
& +\left(\Delta^{4} F^{2} X^{9} M^{-4}\right)^{1 / 10}+X F^{-1 / 4} .
\end{aligned}
$$

$[6]$.

Proof. This is a version of Kolesnik's AB-Theorem. For the proof see 
Lemma 3. Assume that $x$ is a real number with $X^{1 / 2-c+\eta}<|x|<$ $X^{1-c-\eta}$, and that $a(m), b(k)$ are complex numbers of modulus $\leq 1$. Assume further that $M K \asymp X$ and

$$
X^{\eta} \ll K \ll X^{1 / 2} .
$$

Then

$$
\sum_{m \sim M} \sum_{k \sim K} a(m) b(k) e\left(x(m k)^{c}\right) \ll X^{1-\eta / 3} .
$$

Pr o of. Denote the given sum by $U$. We first apply Cauchy's inequality and Weyl's lemma to the sum over $k$ to get

$$
|U|^{2} \ll \frac{X^{2}}{Q}+\frac{X}{Q} \sum_{q \leq Q} \sum_{k \sim K}\left|\sum_{m \sim M} e(f(m))\right| .
$$

Here $f(m)=x\left((k+q)^{c}-k^{c}\right) m^{c}$ and $Q=X^{2 \eta / 3}$. Under the assumptions of the lemma we have

$$
\left|f^{\prime}(m)\right| \asymp|x| q X^{c-1} \ll X^{-\eta / 3} \leq 1 / 2,
$$

so by the Kuz'min-Landau inequality

$$
|U|^{2} \ll|x|^{-1} X^{2-c-\eta / 3} K+X^{2-2 \eta / 3} \ll X^{2-2 \eta / 3} .
$$

Clearly this proves the lemma.

Lemma 4. Assume that $x$ is a real number with $X^{1 / 2-c+\eta}<|x|<$ $X^{1-c-\eta}$, and that $a(m)$ are complex numbers of modulus $\leq 1$. Assume further that $M K \asymp X$ and

$$
K \gg X^{1 / 3+\eta} .
$$

Then

$$
\sum_{m \sim M} \sum_{k \sim K} a(m) e\left(x(m k)^{c}\right) \ll X^{1-\eta / 3} .
$$

Proof. Denote the given sum by $U$. The exponent pair $(1 / 6,2 / 3)$ gives

$$
\begin{aligned}
|U| & \ll M\left(\left(|x| X^{c} K^{-1}\right)^{1 / 6} K^{2 / 3}+\left(|x| X^{c} K^{-1}\right)^{-1}\right) \\
& \ll X\left(X^{1 / 6} K^{-1 / 2}+X^{-1 / 2}\right) \ll X^{1-\eta / 2} .
\end{aligned}
$$

Lemma 5. Let $1<c<61 / 55$. Assume that $x$ is a real number with $X^{1-c-\eta}<|x|<X^{61 / 55-c}$, and that $a(m), b(k)$ are complex numbers of modulus $\leq 1$. Assume further that $M K \asymp X$ and

$$
X^{56 / 165} \ll K \ll X^{123 / 275} .
$$

Then

$$
\sum_{m \sim M} \sum_{k \sim K} a(m) b(k) e\left(x(m k)^{c}\right) \ll X^{49 / 55+\eta} .
$$

Pr o of. This follows immediately from Lemma 4 with $(m, n)=(k, m)$. 
Lemma 6. Let $1<c<61 / 55$. Assume that $x$ is a real number with $X^{1-c-\eta}<|x|<X^{61 / 55-c}$, and that $a(m), b(k)$ are complex numbers of modulus $\leq 1$. Assume further that $M K \asymp X$ and

$$
X^{12 / 55} \ll K \ll X^{844 / 3025} \text {. }
$$

Then

$$
\sum_{m \sim M} \sum_{k \sim K} a(m) b(k) e\left(x(m k)^{c}\right) \ll X^{49 / 55+\eta} .
$$

Pr o of. Denote the given sum by $U$. We start as in Lemma 3 , but we choose a different $Q$, namely $Q=X^{12 / 55}$, and estimate the sum over $m$ in (3.1) using an exponent pair $(\kappa, \lambda)$ rather than the Kuz'min-Landau inequality. Thus,

$$
|U|^{2} \ll(|x| Q)^{\kappa} X^{1+\lambda+\kappa(c-1)} K^{1-\lambda}+X^{98 / 55} \ll X^{98 / 55}
$$

provided that

$$
X^{12 / 55} \ll K \ll X^{1-6(2+3 \kappa) /(55(1-\lambda))} .
$$

Choosing $(\kappa, \lambda)=B A^{2} B A B A B A^{2} B(0,1)=(81 / 242,6 / 11)$, we obtain the result.

Lemma 7. Let $1<c<61 / 55$. Assume that $x$ is a real number with $X^{1-c-\eta}<|x|<X^{61 / 55-c}$, and that $a(m)$ are complex numbers of modulus $\leq 1$. Assume further that $M K \asymp X$ and

$$
K \gg X^{53 / 110} \text {. }
$$

Then

$$
\sum_{m \sim M} \sum_{k \sim K} a(m) e\left(x(m k)^{c}\right) \ll X^{49 / 55+\eta} .
$$

Proof. If $K \gg X^{97 / 165}$, the argument of Lemma 4 proves the desired estimate. If this is not the case, we follow the argument on pp. 123-124 of [5]. Denote the given sum by $U$. Using Cauchy's inequality and Weyl's lemma with $Q=X^{12 / 55}$, we obtain

$$
|U|^{2} \ll \frac{X}{Q} \sum_{q \leq Q} \sum_{m \sim M} \sum_{k \cong K} e\left(x\left((k+q)^{c}-k^{c}\right) m^{c}\right)+X^{98 / 55} .
$$

(Here $k \cong K$ means that $k$ runs through a subinterval of $(K, 2 K]$ which end points may depend on $m$ and $q$.) Denote the sum over $(m, k)$ by $U_{1}(q)$. Applying the Poisson formula and partial summation to $m$ and $k$ successively we find

$$
\left|U_{1}(q)\right| \ll M K F^{-1}\left|\sum_{\mu, \nu} e(f(\mu, \nu))\right|+E
$$

where $F=|x| q X^{c} K^{-1}, \mu \cong F M^{-1}, \nu \cong F K^{-1}$,

$$
f(\mu, \nu) \sim_{q / K} c_{0}(x q)^{1 /(2-2 c)} \nu^{1 / 2} \mu^{c /(2 c-2)} \asymp F
$$


(here $c_{0}$ is a constant depending only on $c$ ), and

$$
E \ll X^{\eta}\left(X F^{-1 / 2}+F X^{-1 / 2}\right) .
$$

Substituting the estimate for $U_{1}(q)$ in (3.5), we get

$$
|U|^{2} \ll \frac{X^{2}}{Q} \sum_{q \leq Q} F^{-1}\left|\sum_{\mu, \nu} e(f(\mu, \nu))\right|+X^{98 / 55} .
$$

Finally, we estimate the sum over $(\mu, \nu)$ in (3.6) using Lemma 2 with $(m, n)$ $=(\mu, \nu)$, if $K \geq X^{1 / 2}$, and with $(m, n)=(\nu, \mu)$, otherwise. After some calculations the result follows.

4. Asymptotic formulas. In this section $\Phi(x)$ is the Fourier transform of the function $\varphi(y)$ defined in Section 1. If $\tau=X^{1-c-\eta}$ and

$$
I_{0}(x)=\int_{X}^{2 X} \frac{e\left(x t^{c}\right)}{\log t} d t
$$

we define

$$
W_{0}(n)=\int_{-\tau}^{\tau} I_{0}^{2}(x) \Phi(x) e\left(\left(n^{c}-N\right) x\right) d x .
$$

Also, while dealing with $\mathcal{A}^{*}$, we will use

$$
I_{1}(x)=\sum_{X^{123 / 275}<p \leq \sqrt{3 X}} \frac{1}{p} \int_{X}^{2 X} \frac{e\left(x t^{c}\right)}{\log p t} d t
$$

and

$$
W_{1}(n)=\int_{-\tau}^{\tau} I_{0}(x) I_{1}(x) \Phi(x) e\left(\left(n^{c}-N\right) x\right) d x
$$

Finally, $X^{\sigma}$ denotes a function of the form $e^{-a(\log X)^{1 / 4}}$ with some unspecified constant $a>0$; in particular, we may write $X^{-\sigma}(\log X)^{A} \ll X^{-\sigma}$ instead of

$$
e^{-a(\log X)^{1 / 4}}(\log X)^{A} \ll e^{-a(\log X)^{1 / 4} / 2} .
$$

Lemma 8. Let $\nu>0$ be fixed, $x \geq x_{0}(\nu), x^{\nu} \leq z \leq x$. Let also $\omega(x)$ be the continuous solution of the differential-difference equation

$$
\begin{cases}\omega(x)=1 / x & \text { if } 1 \leq x \leq 2 \\ (x \omega(x))^{\prime}=\omega(x-1) & \text { if } x>2 .\end{cases}
$$

Then for any $u \in(x, 2 x]$, we have

$$
\sum_{\substack{x<n \leq u \\(n, P(z))=1}} 1=\omega\left(\frac{\log x}{\log z}\right) \cdot \frac{u-x}{\log z}+O\left(\frac{x}{\log ^{2} x}\right) .
$$


Proof. This follows easily from Lemma 2 of [2].

Lemma 9. Let $1<c<61 / 55$. Assume that $a(m), b(k)$ are complex numbers of modulus $\leq 1$. Assume also that $M K \asymp X$ with $K$ satisfying one of the inequalities (3.2) or (3.3). Then

$$
\begin{aligned}
\sum_{m \sim M} \sum_{k \sim K} a(m) b(k) w(m k) & \\
= & \sum_{m \sim M} \sum_{k \sim K} a(m) b(k) W_{0}(m k)+O\left(\varepsilon X^{3-c-\sigma}\right) .
\end{aligned}
$$

Moreover, if $K$ satisfies (3.4),

$$
\sum_{m \sim M} \sum_{k \sim K} a(m) w(m k)=\sum_{m \sim M} \sum_{k \sim K} a(m) W_{0}(m k)+O\left(\varepsilon X^{3-c-\sigma}\right) .
$$

Proof. We consider in detail only (4.3) under the assumption (3.2), since the changes needed in the other cases are obvious. Let $D(X)$ denote the left-hand side of (4.3). By the Fourier inversion formula,

$$
D(X)=\int_{-\infty}^{\infty} S^{2}(x) U(x) \Phi(x) e(-N x) d x
$$

where

$$
S(x)=\sum_{p \sim X} e\left(x p^{c}\right), \quad U(x)=\sum_{m \sim M} \sum_{k \sim K} a(m) b(k) e\left(x(m k)^{c}\right) .
$$

We set $\tau=X^{1-c-\eta}$ and $H=\varepsilon^{-1} \log ^{2} X$, and define the sets

$$
\begin{gathered}
E_{1}=\{x \in \mathbb{R}:|x|<\tau\}, \quad E_{2}=\{x \in \mathbb{R}: \tau \leq|x| \leq H\}, \\
E_{3}=\{x \in \mathbb{R}:|x|>H\} .
\end{gathered}
$$

From (1.4) and the trivial estimates for $S(x)$ and $U(x)$ we find

$$
\begin{aligned}
D_{3}(X) & =\int_{E_{3}} S^{2}(x) U(x) \Phi(x) e(-N x) d x \\
& \ll X^{3}\left(\frac{r}{2 \pi \Delta}\right)^{r} \int_{H}^{\infty} x^{-(r+1)} d x \ll 1 .
\end{aligned}
$$

Now, for $x \in E_{2}$ and $K$ satisfying (3.2), Lemma 5 provides the estimate

$$
\max _{x \in E_{2}}|U(x)| \ll X^{49 / 55+\eta} .
$$

(If $K$ satisfies (3.3) or (3.4), we refer to Lemmas 6 or 7, respectively.) Also it is easy to prove (see for example Lemma 7 of [10]) that for any integer $n$,

$$
\int_{n}^{n+1}|S(x)|^{2} d x \ll X .
$$


Using the last two inequalities and (1.4), we obtain

$$
\begin{aligned}
D_{2}(X) & =\int_{E_{2}} S^{2}(x) U(x) \Phi(x) e(-N x) d x \\
& \ll \varepsilon X^{49 / 55+\eta} \int_{0}^{1}|S(x)|^{2} d x+X^{49 / 55+\eta} \sum_{n \leq H} \frac{1}{n} \int_{n}^{n+1}|S(x)|^{2} d x \\
& \ll \varepsilon X^{104 / 55+\eta}+X^{104 / 55+\eta} \log X \ll \varepsilon X^{3-c-\eta} .
\end{aligned}
$$

Finally, consider

$$
D_{1}(X)=\int_{E_{1}} S^{2}(x) U(x) \Phi(x) e(-N x) d x .
$$

For $x \in E_{1}$, the argument on pp. 301-303 of [10] establishes the asymptotic formula

$$
S(x)=I_{0}(x)+O\left(X^{1-\sigma}\right) .
$$

Also, following the argument of Lemma 7 of [10], we have the estimate

$$
\int_{-\tau}^{\tau}|U(x)|^{2} d x \ll X^{2-c} \log ^{4} X
$$

and similar (and even better) upper bounds for the corresponding means of $S(x)$ and $I_{0}(x)$. Hence,

$$
\begin{aligned}
D_{1}(X) & =\int_{-\tau}^{\tau} I_{0}^{2}(x) U(x) \Phi(x) e(-N x) d x+O\left(\varepsilon X^{3-c-\sigma}\right) \\
& =\sum_{m \sim M} \sum_{k \sim K} a(m) b(k) W_{0}(m k)+O\left(\varepsilon X^{3-c-\sigma}\right) .
\end{aligned}
$$

The lemma follows from (4.5)-(4.9).

The next result is the $\mathcal{A}^{*}$-version of Lemma 9 .

Lemma 10. Let $1<c<61 / 55$. Assume that $a(m), b(k)$ are complex numbers of modulus $\leq 1$. Assume also that $M K \asymp X$ with $K$ satisfying one of the inequalities (3.2) or (3.3). Then

$$
\begin{aligned}
\sum_{m \sim M} \sum_{k \sim K} a(m) b & (k) w^{*}(m k) \\
= & \sum_{m \sim M} \sum_{k \sim K} a(m) b(k) W_{1}(m k)+O\left(\varepsilon X^{3-c-\sigma}\right) .
\end{aligned}
$$

Moreover, if $K$ satisfies (3.4),

$$
\sum_{m \sim M} \sum_{k \sim K} a(m) w^{*}(m k)=\sum_{m \sim M} \sum_{k \sim K} a(m) W_{1}(m k)+O\left(\varepsilon X^{3-c-\sigma}\right) .
$$


Pro of. The argument is similar to the one used in the proof of Lemma 9. If the sum under consideration is $D^{*}(X)$, we have

$$
D^{*}(X)=\int_{-\infty}^{\infty} S(x) S_{1}(x) U(x) \Phi(x) e(-N x) d x
$$

where $S(x)$ and $U(x)$ are the same as before, and

$$
S_{1}(x)=\sum_{\substack{p q \sim X \\ X^{123 / 275}<p \leq q}} e\left(x(p q)^{c}\right) .
$$

The proof of the inequality

$$
\int_{|x|>\tau} S(x) S_{1}(x) U(x) \Phi(x) e(-N x) d x \ll \varepsilon X^{3-c-\eta}
$$

repeats verbatim the estimates of $D_{2}(X)$ and $D_{3}(X)$ from Lemma 9 , so we can concentrate on

$$
\int_{-\tau}^{\tau} S(x) S_{1}(x) U(x) \Phi(x) e(-N x) d x
$$

As in the proof of Lemma 9 , we can replace $S(x)$ by $I_{0}(x)$. Thus, the integral (4.12) equals

$$
\int_{-\tau}^{\tau} I_{0}(x) S_{1}(x) U(x) \Phi(x) e(-N x) d x+O\left(\varepsilon X^{3-c-\sigma}\right) .
$$

However, we cannot do the same with $S_{1}(x)$. The reason is that the approach from [10] establishes the asymptotic formula

$$
S_{1}(x)=I_{1}(x)+O\left(X^{1-\sigma}\right)
$$

only for $|x| \leq \tau_{1}=X^{152 / 275-c-\eta}$. Fortunately, we can go around this by showing that the values of $x$ with $\tau_{1}<|x|<\tau$ do not contribute much to (4.13). For, this portion of the integral is

$$
\begin{aligned}
& \ll \varepsilon \max _{\tau_{1}<|x|<\tau}|U(x)|\left(\int_{-\tau}^{\tau}\left|S_{1}(x)\right|^{2} d x+\int_{-\tau}^{\tau}\left|I_{0}(x)\right|^{2} d x\right) \\
& \ll \max _{\tau_{1}<|x|<\tau}|U(x)| \varepsilon X^{2-c} \log X \ll \varepsilon X^{3-c-\eta / 4}
\end{aligned}
$$

by virtue of Lemma 3. Hence, the integral (4.12) equals

$$
\begin{aligned}
\int_{-\tau_{1}}^{\tau_{1}} I_{0}(x) I_{1}(x) U(x) & \Phi(x) e(-N x) d x+O\left(\varepsilon X^{3-c-\sigma}\right) \\
= & \int_{-\tau}^{\tau} I_{0}(x) I_{1}(x) U(x) \Phi(x) e(-N x) d x+O\left(\varepsilon X^{3-c-\sigma}\right) .
\end{aligned}
$$


Clearly the above discussion proves (4.10). The proof of (4.11) is similar with Lemma 4 replacing Lemma 3.

Lemma 11. Let $1<c<61 / 55, M K \asymp X$, and $K$ satisfies one of the inequalities (3.2) or (3.3). Let $I, J$ be integers and $\mathcal{I}_{i}, \mathcal{J}_{j}$ are intervals for $1 \leq i \leq I, 1 \leq j \leq J$. Write

$$
a(m, k)=\sum_{\substack{r p_{1} \ldots p_{I}=k \\ p_{1}<\ldots<p_{I} \\ p_{i} \in \mathcal{I}_{i}}} c(k) \sum_{\substack{l q_{1} \ldots q_{J}=m \\ q_{1}<\ldots<q_{J} \\ q_{j} \in \mathcal{J}_{j}}} d(m)
$$

with $|c(k)|,|d(m)| \leq 1$ and $p_{1}, \ldots, p_{I}$ and $q_{1}, \ldots, q_{J}$ satisfying $O(1)$ joint conditions of the form

$$
p_{u} \leq q_{v} \quad \text { or } \quad q_{v} \leq p_{u}
$$

or

$$
\prod_{u \in \mathcal{U}} p_{u} \prod_{v \in \mathcal{V}} q_{v} \leq H \quad \text { or } \quad \prod_{u \in \mathcal{U}} p_{u} \geq \prod_{v \in \mathcal{V}} q_{v}
$$

or similar $($ for given $\mathcal{U} \subset\{1, \ldots, I\}, \mathcal{V} \subset\{1, \ldots, J\}, H \leq X)$. Then

$$
\sum_{m \sim M} \sum_{k \sim K} a(m, k) w(m k)=\sum_{m \sim M} \sum_{k \sim K} a(m, k) W_{0}(m k)+O\left(\varepsilon X^{3-c-\sigma}\right) .
$$

Furthermore, the result still holds if we replace $w(n)$ by $w^{*}(n)$ and $W_{0}(n)$ by $W_{1}(n)$.

Proof. We follow the approach from Lemma 1 of [4]: we first remove the dependencies between the variables $m$ and $k$, and then refer to (4.3). Each joint condition can be removed via Perron's formula

$$
\frac{1}{\pi} \int_{-T}^{T} e^{i \gamma t} \frac{\sin \beta t}{t} d t= \begin{cases}1+O\left(T^{-1}(\beta-|\gamma|)^{-1}\right) & \text { if }|\gamma| \leq \beta \\ O\left(T^{-1}(|\gamma|-\beta)^{-1}\right) & \text { if }|\gamma|>\beta\end{cases}
$$

at the cost of an extra $\log X$ factor in the error term. For instance, if we have a single condition $p_{u}<q_{v}$, we take $\gamma=\log p_{u}, \beta=\log \left(q_{v}+1 / 2\right)$, and $T=X^{2}$, and get

$$
\sum_{m \sim M} \sum_{k \sim K} a(m, k) w(m k)=\frac{1}{\pi} \int_{-T}^{T} \sum_{m \sim M} \sum_{k \sim K} a(m, k, t) w(m k) \frac{d t}{t}+O\left(\varepsilon X^{3-c-\eta}\right)
$$

where $a(m, k, t)$ is defined as $a(m, k)$ but with the factors $p_{u}^{i t}, \sin \left(t\left(\log \left(q_{v}+\right.\right.\right.$ $1 / 2))$ ) included, and the condition $p_{u}<q_{v}$ removed. Hence, we can rewrite $a(m, k, t)$ as $a^{*}(m, t) b^{*}(k, t)$, and then refer to (4.3) to obtain 


$$
\begin{array}{r}
\frac{1}{\pi} \int_{-T}^{T} \sum_{m \sim M} \sum_{k \sim K} a^{*}(m, t) b^{*}(k, t) W_{0}(m k) \frac{d t}{t}+O\left(\varepsilon X^{3-c-\sigma} \log X\right) \\
=\sum_{m \sim M} \sum_{k \sim K} a(m, k) W_{0}(m k)+O\left(\varepsilon X^{3-c-\sigma}\right)
\end{array}
$$

after another application of Perron's formula. Obviously, if we have more joint conditions, say $A$, we will end up with an $A$-tuple integral and a $(\log X)^{A}$ factor in the error term.

Before going further we define the integrals

$$
\begin{aligned}
I(x) & =\int_{X}^{2 X} e\left(x t^{c}\right) d t, \\
J_{0}(X) & =\int_{-\infty}^{\infty} I_{0}^{2}(x) I(x) \Phi(x) e(-N x) d x, \\
J_{1}(X) & =\int_{-\infty}^{\infty} I_{0}(x) I_{1}(x) I(x) \Phi(x) e(-N x) d x .
\end{aligned}
$$

LEMMA 12. Let $1<c<61 / 55$ and $u \geq 1$, and for some $K$ satisfying one of the conditions (3.2) or (3.3) there exists a $\mathcal{D} \subset\{1, \ldots, u\}$ with

$$
\prod_{j \in \mathcal{D}} p_{j} \sim K
$$

Then

$$
\begin{array}{rl}
\sum_{p_{1}, \ldots, p_{u}} & S\left(\mathcal{A}_{p_{1} \ldots p_{u}}, p_{1}\right) \\
= & J_{0}(X) \sum_{p_{1}, \ldots, p_{u}} \frac{S\left(\mathcal{B}_{p_{1} \ldots p_{u}}, p_{1}\right)}{p_{1} \ldots p_{u}}+O\left(\varepsilon X^{3-c}(\log X)^{-10 / 3}\right) .
\end{array}
$$

Here the summation is over primes $p_{1}, \ldots, p_{u} \geq X^{89 / 825}$ satisfying $p_{j}>p_{1}$, together with $O(1)$ further conditions of the type

$$
p_{j} \leq p_{l} \quad \text { or } \quad Q \leq \prod_{j \in \mathcal{F}} p_{j} \leq R
$$

for some $\mathcal{F} \subset\{1, \ldots, u\}$ and $R \leq X$. Also,

$$
\begin{array}{rl}
\sum_{p_{1}, \ldots, p_{u}} & S\left(\mathcal{A}_{p_{1} \ldots p_{u}}^{*}, p_{1}\right) \\
= & J_{1}(X) \sum_{p_{1}, \ldots, p_{u}} \frac{S\left(\mathcal{B}_{p_{1} \ldots p_{u}}, p_{1}\right)}{p_{1} \ldots p_{u}}+O\left(\varepsilon X^{3-c}(\log X)^{-10 / 3}\right) .
\end{array}
$$

The result still holds if, instead of $K, X / K$ satisfies (3.2) or (3.3). 
Pr o of. The left-hand side of (4.15) equals

$$
\sum_{p_{1}, \ldots, p_{u}} \sum_{\substack{n \sim X /\left(p_{1} \ldots p_{u}\right) \\\left(n, P\left(p_{1}\right)\right)=1}} w\left(p_{1} \ldots p_{u} n\right) .
$$

Setting

$$
k=\prod_{j \in \mathcal{D}} p_{j} \quad \text { and } \quad m=\left(\prod_{j \notin \mathcal{D}} p_{j}\right) \cdot n
$$

we can represent the last sum in the form appearing on the left side of (4.14). So, it equals

$$
\int_{-\tau}^{\tau} I_{0}^{2}(x) U(x) \Phi(x) e(-N x) d x+O\left(\varepsilon X^{3-c-\sigma}\right)
$$

where

$$
U(x)=\sum_{p_{1}, \ldots, p_{u}} \sum_{\substack{n \sim X /\left(p_{1} \ldots p_{u}\right) \\\left(n, P\left(p_{1}\right)\right)=1}} e\left(x\left(p_{1} \ldots p_{u} n\right)^{c}\right) .
$$

Since, by the first derivative estimate for trigonometric integrals (see Lemma 1 on p. 47 of [7]),

$$
\left|I_{0}(x)\right| \ll \frac{1}{|x| X^{c-1} \log X},
$$

and

$$
|U(x)| \ll \frac{X}{\log X},
$$

the values of $x$ with $|x| \geq \tau_{1}=X^{-c}(\log X)^{1 / 3}$ contribute to the last integral at most $O\left(\varepsilon X^{3-c}(\log X)^{-10 / 3}\right)$. On the other hand, if $|x|<\tau_{1}$, Lemma 8 and partial summation imply that

$$
U(x)=I(x) \sum_{p_{1}, \ldots, p_{u}} \frac{S\left(\mathcal{B}_{p_{1} \ldots p_{u}}, p_{1}\right)}{p_{1} \ldots p_{u}}+O\left(X(\log X)^{-5 / 3}\right) .
$$

Combining the above estimates completes the proof.

LeMma 13. Let $1<c<61 / 55$ and $M \leq X^{123 / 275}$. Suppose further that $a(m)$ are real numbers such that $a(m) \ll 1$ and $a(m)=0$ unless all prime divisors of $m$ are $\geq X^{89 / 825}$. Then

$$
\begin{aligned}
& \sum_{m \sim M} a(m) S\left(\mathcal{A}_{m}, X^{89 / 825}\right) \\
& =J_{0}(X) \sum_{m \sim M} \frac{a(m)}{m} S\left(\mathcal{B}_{m}, X^{89 / 825}\right)+O\left(\varepsilon X^{3-c}(\log X)^{-10 / 3}\right) .
\end{aligned}
$$


Also

$$
\begin{aligned}
& \sum_{m \sim M} a(m) S\left(\mathcal{A}_{m}^{*}, X^{89 / 825}\right) \\
& =J_{1}(X) \sum_{m \sim M} \frac{a(m)}{m} S\left(\mathcal{B}_{m}, X^{89 / 825}\right)+O\left(\varepsilon X^{3-c}(\log X)^{-10 / 3}\right) .
\end{aligned}
$$

Pr o of. We shall use the Eratosthenes-Legendre sieve, which states that

$$
\sum_{\substack{n \leq x \\(n, P(z))=1}} f(n)=\sum_{\substack{n d \leq x \\ d \mid P(z)}} \mu(d) f(n d)
$$

where $\mu(d)$ is the Möbius function.

We choose $z=X^{89 / 825}$ and, applying (4.19) to $S\left(\mathcal{A}_{m}, z\right)$, we find

$$
\sum_{m \sim M} a(m) S\left(\mathcal{A}_{m}, z\right)=\sum_{m \sim M} \sum_{\substack{n d \sim X / m \\ d \mid P(z)}} a(m) \mu(d) w(m n d) .
$$

Now we proceed to show that

$$
\begin{aligned}
\sum_{m \sim M} \sum_{\substack{n d \sim X / m \\
d \mid P(z)}} a(m) \mu(d) w(m n d) & \\
= & \sum_{m \sim M} \sum_{\substack{n d \sim X / m \\
d \mid P(z)}} a(m) \mu(d) W_{0}(m n d)+O\left(\varepsilon X^{3-c-\sigma}\right) \\
& =\sum_{m \sim M} \sum_{\substack{n \sim X / m \\
(n, P(z))=1}} a(m) W_{0}(m n)+O\left(\varepsilon X^{3-c-\sigma}\right)
\end{aligned}
$$

after another application of (4.19).

If $M \geq X^{56 / 165}$, we produce a new variable $k=d n$ and derive (4.21) from (4.3). Now suppose that $M \leq X^{56 / 165}$. Then we divide the sum on the left-hand side of (4.21) into two parts: $\sum_{1}$ in which $m d \leq X^{123 / 275}$, and $\sum_{2}$ in which $m d>X^{123 / 275}$. To obtain asymptotic formula for $\sum_{1}$ we combine $m$ and $d$ into a new variable $k$ and refer to (4.4). Then we turn our attention to $\sum_{2}$. It can be written in the form

$$
-\sum_{m \sim M} \sum_{p<z} \sum_{\begin{array}{c}
n p d \sim X / m \\
d \mid P(p) \\
m p d>X^{123 / 275}
\end{array}} a(m) \mu(d) w(m n p d) .
$$

Let $\sum_{3}$ be the part of this sum with $m d \leq X^{123 / 275}<m p d$, and $\sum_{4}$ the part with $m d>X^{123 / 275}$. Introducing the variables $k=m d$ and $l=p n$, we can put $\sum_{3}$ in the form appearing on the left-hand side of (4.14) and then refer 
to Lemma 11 to get the desired asymptotic formula (since $m p d>X^{123 / 275}$ and $p<z$, we must have $m d>X^{56 / 165}$ ). Again, $\sum_{4}$ can be rewritten as

$$
\sum_{m \sim M} \sum_{p_{1}<p_{2}<z} \sum_{\substack{n p_{1} p_{2} d \sim X / m \\ d \mid P\left(p_{1}\right) \\ m p_{1} d>X^{123 / 275}}} a(m) \mu(d) w\left(m n p_{1} p_{2} d\right) ;
$$

then we can use Lemma 11 to find an asymptotic formula for the part of the last sum with $m d<X^{123 / 275}$, and can proceed further with the rest of it. We can continue in this fashion, obtaining at each step a sum to which Lemma 11 applies and a sum for which further decomposition can be given. Since every integer $\leq 2 X$ has at most $O(\log X)$ prime divisors, after $\ll \log X$ steps, this procedure will stop and we will be left with a sum which does not require further decomposition (Lemma 11 applies to all of it). Combining the asymptotic formulas for all the occurring sums, we complete the proof of (4.21).

So, using (4.20) and (4.21), we get

$$
\sum_{m \sim M} a(m) S\left(\mathcal{A}_{m}, z\right)=\sum_{m \sim M} \sum_{\substack{n \sim X / m \\(n, P(z))=1}} a(m) W_{0}(m n)+O\left(\varepsilon X^{3-c-\sigma}\right) .
$$

Also, using the approach from the proof of the previous lemma, we have

$$
\begin{aligned}
& \sum_{m \sim M} \sum_{\substack{n \sim X / m \\
(n, P(z))=1}} a(m) W_{0}(m n) \\
& \quad=J_{0}(X) \sum_{m \sim M} \frac{a(m)}{m} S\left(\mathcal{B}_{m}, z\right)+O\left(\varepsilon X^{3-c}(\log X)^{-10 / 3}\right) .
\end{aligned}
$$

The result follows from (4.22) and (4.23).

5. Proof of Theorem 1. We start with (2.4). We can estimate $S_{1}, S_{7}$, $S_{12}, S_{1}^{*}$, and $S_{7}^{*}$ using Lemma 13. Consider, for example, $S_{12}$. By (4.17), it equals (we use the values of $A, B, C, D$, and $F$ from Section 2)

$$
J_{0}(X) \sum_{C<p<D} \frac{1}{p} \sum_{\substack{p n \sim X \\(n, P(A))=1}} 1+O\left(\varepsilon X^{3-c}(\log X)^{-10 / 3}\right) .
$$

Using Lemma 8 and partial summation, we infer that the sum over $p$ and $n$ is

$$
\frac{825}{89 \log X} \int_{844 / 3025}^{56 / 165} \omega\left(\frac{1-x}{89 / 825}\right) \frac{d x}{x}+O\left((\log X)^{-2}\right) \leq \frac{1.0200}{\log X}+O\left((\log X)^{-2}\right) .
$$


We also have

$$
\begin{aligned}
J_{0}(X) & =\frac{1}{\log ^{2} X} \int_{-\infty}^{\infty} I^{3}(x) \Phi(x) e(-N x) d x+O\left(\varepsilon X^{3-c}(\log X)^{-3}\right) \\
& =: \frac{J(X)}{\log ^{2} X}+O\left(\varepsilon X^{3-c}(\log X)^{-3}\right) .
\end{aligned}
$$

Hence,

$$
S_{12} \leq 1.0200 \frac{J(X)}{\log ^{3} X}+O\left(\varepsilon X^{3-c}(\log X)^{-10 / 3}\right) .
$$

We can evaluate the remaining four quantities similarly. Introducing the notation $S_{12} \lesssim 1.0200$ as a shortcut for the last inequality, we can state the corresponding estimates as

$$
S_{1} \gtrsim 5.2039, \quad S_{7} \lesssim 3.6666, \quad S_{1}^{*} \lesssim 1.1017, \quad S_{7}^{*} \gtrsim 0.7762,
$$

where we have used the fact that

$$
\begin{aligned}
J_{1}(X) & =\frac{J(X)}{\log ^{2} X} \int_{123 / 275}^{1 / 2} \frac{d x}{x(1-x)}+O\left(\varepsilon X^{3-c}(\log X)^{-3}\right) \\
& =\frac{J(X)}{\log ^{2} X} \ln \left(\frac{152}{123}\right)+O\left(\varepsilon X^{3-c}(\log X)^{-3}\right) .
\end{aligned}
$$

(This follows easily from the Prime Number Theorem.) Further, we obviously have

$$
S_{8}^{*} \leq \sum_{\substack{A \leq q<p<B \\ p q<B}} S\left(\mathcal{A}_{p q}^{*}, A\right) \quad \text { and } \quad S_{10}^{*} \leq \sum_{\substack{A \leq q<p<B \\ C<p q<D}} S\left(\mathcal{A}_{p q}^{*}, A\right),
$$

and these sums can also be estimated via Lemma 13. Hence,

$$
S_{8}^{*} \lesssim 0.0002 \text { and } S_{10}^{*} \lesssim 0.1290 \text {. }
$$

Now consider $S_{9}^{*}$. By Lemma 12, it equals

$$
J_{1}(X) \sum_{\substack{A \leq q<p<B \\ B \leq p q \leq C}} \frac{1}{p q} \sum_{\substack{p q n \sim X \\(n, P(q))=1}} 1+O\left(\varepsilon X^{3-c}(\log X)^{-10 / 3}\right) .
$$

As before, using Lemma 8 and partial summation, we can evaluate the sum over $p, q$, and $n$. We have

$$
S_{9}^{*} \lesssim 0.0619
$$

and similarly,

$$
\begin{aligned}
& S_{3} \lesssim 0.5632, \quad S_{5} \lesssim 0.4544, \quad S_{9} \gtrsim 0.2923, \quad S_{11} \gtrsim 0.2369, \\
& S_{14} \gtrsim 0.1108, \quad S_{3}^{*} \gtrsim 0.1192, \quad S_{5}^{*} \gtrsim 0.0961, \quad S_{11}^{*} \lesssim 0.0502 .
\end{aligned}
$$


Also, we can use Lemma 13 to evaluate the parts of $S_{13}$ with $D \leq p q \leq F$ and $X / F \leq p q \leq X / D$, and the part of $S_{15}$ with $X / F \leq p q \leq X / D$. Hence, we have

$$
S_{13} \gtrsim 0.2157 \text { and } S_{15} \gtrsim 0.0480 .
$$

Substituting all these estimates in (2.4), we find

$$
S\left(\mathcal{A},(3 X)^{1 / 2}\right) \geq 0.0519 \frac{J(X)}{\log ^{3} X}+O\left(\varepsilon X^{3-c}(\log X)^{-10 / 3}\right) .
$$

Since, by Lemma 6 of [10], $J(X) \gg \varepsilon X^{3-c}$, this inequality establishes (2.1) and completes the proof of the theorem.

6. Proof of Theorem 2. Fix $\varrho>0$ and consider the set

$$
\mathcal{E}_{\varrho}(X)=\left\{c \in(1+\varrho, 3 / 2-\varrho):\left|R^{*}(N, c)-J^{*}(N, c)\right| \geq X^{3 / 2} \log ^{2} X\right\}
$$

where

$$
\begin{aligned}
& R^{*}(N, c)=\sum_{p_{1}, p_{2}, p_{3} \sim X} \varphi\left(p_{1}^{c}+p_{2}^{c}+p_{3}^{c}-N\right), \\
& J^{*}(N, c)=\int_{-\infty}^{\infty} I_{0}^{3}(x) \Phi(x) e(-N x) d x .
\end{aligned}
$$

Note that by Lemma 6 of [10],

$$
J^{*}(N, c) \gg X^{3 / 2} \log ^{7} X,
$$

so we can deduce Theorem 2 by showing that the Lebesgue measure of $\mathcal{E}_{\varrho}(X)$ is $O\left(\log ^{-1} X\right)$ (hereafter $X \geq X_{0}(\varrho)$ and the implied constants depend at most on $\varrho$ ). This follows from the estimate

$$
\int_{1+\varrho}^{3 / 2-\varrho}\left(R^{*}(N, c)-J^{*}(N, c)\right)^{2} d c \ll X^{3} \log ^{3} X
$$

by an application of Chebyshev's inequality. Hence, it suffices to show that

$$
\int_{a}^{b}\left(R^{*}(N, c)-J^{*}(N, c)\right)^{2} d c \ll X^{3} \log ^{2} X
$$

whenever $1+\varrho \leq a \leq 3 / 2-\varrho$ and $b=a+\log ^{-1} X$.

The framework for the proof of (6.1) is the same as for the proof of Lemma 9. We have

$$
R^{*}(N, c)=\int_{-\infty}^{\infty} S^{3}(x, c) \Phi(x) e(-N x) d x
$$

where $S(x, c)=S(x)$ is the exponential sum used in Section 4. Upon setting $\eta=\varrho^{2}$, we can define (at least formally) the parameters $\tau$ and $H$, and the sets $E_{1}, E_{2}$, and $E_{3}$ as in Lemma 9 . Then $R^{*}(N, c)$ can be written as the 
sum of three integrals corresponding to $E_{1}, E_{2}$, and $E_{3}$, respectively. One can easily check that similarly to the estimate of $D_{3}(X)$ in the proof of Lemma 9 we have

$$
\int_{E_{3}}\left|S^{3}(x) \Phi(x)\right| d x \ll 1
$$

uniformly with respect to $c \in(1,3 / 2)$. Also, the argument used to evaluate $D_{1}(X)$ in that proof (see also pp. 305-306 in [10]) shows that uniformly in $c$

$$
\begin{aligned}
\int_{E_{1}} S^{3}(x) \Phi(x) e(-N x) d x & =J^{*}(N, c)+O\left(\varepsilon X^{3-c}(\log X)^{-20}\right) \\
& =J^{*}(N, c)+O\left(X^{3 / 2}(\log X)^{-10}\right) .
\end{aligned}
$$

Thus, (6.1) will follow if we prove that

$$
D(X):=\int_{a}^{b}\left(\int_{E_{2}}\left|S^{3}(x, c) \Phi(x)\right| d x\right)^{2} d c \ll X^{3} \log ^{2} X .
$$

By (1.4) and Cauchy's inequality, we obtain that

$$
D(X) \ll \int_{a}^{b} \varepsilon^{2}(c)\left(\int_{E_{2}}|S(x, c)|^{2} d x\right)\left(\int_{E_{2}}|S(x, c)|^{4} d x\right) d c .
$$

Since the estimate (4.7) is uniform in $n$ and $c>1$, we find that

$$
\int_{E_{2}}|S(x, c)|^{2} d x \ll X H(c)=\varepsilon^{-1}(c) X \log ^{2} X,
$$

and hence,

$$
\begin{aligned}
D(X) & \ll X \log ^{2} X \int_{a}^{b} \varepsilon(c)\left(\int_{E_{2}}|S(x, c)|^{4} d x\right) d c \\
& \ll \varepsilon(b) X \log ^{2} X \int_{\tau(b)}^{H(a)} \int_{a}^{b}|S(x, c)|^{4} d c d x \\
& \ll \varepsilon(b) X \log ^{2} X \int_{\tau(b)}^{H(a)}\left|S\left(x, c_{0}\right)\right|^{2} \int_{a}^{b}|S(x, c)|^{2} d c d x
\end{aligned}
$$

for some $c_{0} \in[a, b]$.

Next, we need an estimate for the inner integral. The one we use is

$$
\int_{a}^{b}|S(x, c)|^{2} d c \ll \frac{X}{\log ^{2} X}+\frac{X^{2-a}}{|x| \log X} .
$$


(It can be proved via the approach from Lemma 7 of [10]; note that the integration variable is $c$.) This estimate, (4.7), and (6.3) imply

$$
D(X) \ll \varepsilon(b) X \log ^{2} X\left\{\frac{H(a) X^{2}}{\log ^{2} X}+\frac{X^{2-a}}{\tau(b) \log X}+X^{3-a}\right\} .
$$

Observe that $X^{b}=e X^{a}$, and hence, for example, $H(a) \asymp H(b)$. Therefore, the last inequality establishes (6.2) and completes the proof of Theorem 2.

\section{References}

[1] Y. C. Cai, On a diophantine inequality involving prime numbers, Acta Math. Sinica 39 (1996), 733-742 (in Chinese).

[2] J. B. Friedlander, Integers free from large and small primes, Proc. London Math. Soc. (3) 33 (1976), 565-576.

[3] G. Harman, On the distribution of $\alpha p$ modulo one, J. London Math. Soc. (2) 27 (1983), 9-18.

[4] - On the distribution of ap modulo one II, Proc. London Math. Soc. (3) 72 (1996), 241-260.

[5] A. Kumchev and T. Nedeva, On an equation with prime numbers, Acta Arith. 83 (1998), 117-126.

[6] H.-Q. Liu, On square-full numbers in short intervals, Acta Math. Sinica (N.S.) 65 (1993), 148-164.

[7] H. L. Montgomery, Ten Lectures on the Interface Between Analytic Number Theory and Harmonic Analysis, CBMS Regional Conf. Ser. in Math., Amer. Math. Soc., Providence, R.I., 1994.

[8] I. I. Piatetski-Shapiro, On a variant of the Waring-Goldbach problem, Mat. Sb. 30 (1952), 105-120 (in Russian).

[9] P. Sargos and J. Wu, Multiple exponential sums with monomials and their applications in number theory, preprint, 1997.

[10] D. I. Tolev, On a diophantine inequality involving prime numbers, Acta Arith. 61 (1992), 289-306.

[11] I. M. Vinogradov, Representation of an odd number as the sum of three primes, Dokl. Akad. Nauk SSSR 15 (1937), 291-294 (in Russian).

Department of Mathematics

University of South Carolina

Columbia, SC 29208

U.S.A.

E-mail: koumtche@math.sc.edu 